\title{
Biosocial spaces and neurocomputational governance: brain-based and brain-targeted technologies in education
}

\author{
Ben Williamson, University of Stirling, UK, ben.williamson@stir.ac.uk \\ Jessica Pykett, University of Birmingham, UK \\ Selena Nemorin, London School of Economics, UK
}

[Pre-publication version of paper accepted in Discourse: Studies in the cultural politics of education, special issue on 'New biological rationalities in education']

Abstract Recently, technologies based on neuroscientific insights into brain function and structure have been promoted for application in education. The novel practices and environments produced by these technologies require new forms of 'biosocial' analysis to unpack their implications for education, learning and governance. The article provides an original analysis of current 'brain-based' R\&D by the edu-business Pearson to apply artificial intelligence in education, and by the computing company IBM to develop cognitive systems for learning. These emerging forms of neurocomputation are examined as technologies designed to function according to neuroscientific understandings of the brain, and to impress themselves on the cerebral lives of learners by being embedded in educational spaces. To examine the technological and neurobiological means by which a learner is made up through technologically-mediated educational environments, we advance the idea of 'brain/code/space' as a conceptual framework. This describes environments that possess brain-like functions of learning and cognition performed by computational processes. The brain/code/spaces of education proposed by Pearson and IBM are intended to optimize human cognition as a technique of human capital development in order to enhance the performance of education systems to secure comparative advantage in a globalizing policy space, exemplifying new forms of neurocomputational governance and capital.

Keywords artificial intelligence, big data, biosocial, brain, cognitive computing, neuroscience

Technologies inspired by the human brain are becoming a pervasive presence in everyday life. Machine learning, neural networks algorithms, cognitive computing systems and even forms of artificial intelligence based on insights from neuroscience already power search engines, social media platforms, and online consumer services (Rose et al 2016). Advances in 'neurotechnology' innovation are also promising new brain-computer interfaces, cognitive training tools and electronic neurostimulators that in coming decades may become 'increasingly effective in modulating the neural correlates of our psychology and behaviour' (Ienca \& Andorno 2017: 5). Recently, similar digital technologies inspired by neuroscience have been promoted for application in education. The novel practices and environments produced by these technologies require new forms of analysis in order to unpack their 
implications for education, learning and governance. Brain-based forms of 'neurocomputation,' we argue in this article, can be understood as 'biosocial' technologies designed to function according to neuroscientific understandings of the plasticity of the brain and biological understandings of epigenetics, and are intended to impress themselves on the cerebral lives of learners.

Taking up a critical biosocial approach to education that acknowledges the reciprocal connections between biological and social processes (Youdell 2016a), this paper focuses on two recent developments in brain-based technologies in education. The first is $R \& D$ conducted by Pearson, the world's largest education business, to apply artificial intelligence to education (AIEd). Its claims, we argue, emphasize the role of biosocial technologies in the performativity of education systems, as brain-inspired technologies that seek to optimize human capital development by enacting real-time measurement at large scale. The second area is cognitive computing undertaken by the global computing firm IBM. IBM is extending its cognitive computing ambitions towards education in the shape of 'bio-mimetic' cognitive learning systems which, it claims, emulate the brain to enable people and machines to interact more 'naturally', extending human cognition itself. IBM's cognitive computing R\&D depends on the translation of biological neural networks studied by neuroscientists into the artificial neural networks applied in cognitive computing and AI design-neural networks that are inspired by biological processes and approximated by mathematical operations. IBM mobilizes biosocial framings of human neuroplasticity and epigenetics both as an inspiration for its cognitive systems and as a means to enhance human cognition. In 2016, the two organizations announced a global partnership to begin embedding IBM's cognitive systems in Pearson's digital courseware.

However, these developments raise serious questions about how the brain is being modelled computationally in bio-mimetic systems, or how AI and cognitive systems developments might interact with human actors to reshape subjectivities. To examine the means by which a learner is made up through technologically- and neurobiologically-mediated educational environments, we advance the idea of 'brain/code/space' as a conceptual framework (Williamson 2017). This integration of geographical research on 'brain culture' (Pykett 2015) and technologically-augmented 'code/space' (Kitchin \& Dodge 2011) describes environments that possess brain-like functions of learning and cognition performed by computational processes. It is argued that the work of global technology and publishing companies such as Pearson and IBM may have the effect of transforming educational 
environments into biosocial brain/code/spaces that are simultaneously brain-based and braintargeted. These brain/code/spaces reframe learning and educational performance as a means for consumers of new educational products and services to secure comparative advantage within a globally competitive educational market. As such they exemplify the emergence of new forms of neurocomputational governance and capital which have the potential to reshape education and learner identities.

\section{Critical biosocial studies}

Biosocial studies emphasize how social environments are now understood to influence the biological functions of the body and even the brain. Critical sociological studies have drawn attention in particular to how 'the brain is a multiply connected device profoundly shaped by social influences,' and how 'the body bears the inscriptions of its socially and materially situated milieu' (Meloni et al 2016: 9, 13). Biological concepts such as 'neuroplasticity' and 'epigenetics' are key here. Neuroplasticity recognizes that the brain is constantly adapting to external stimuli and social environments, while epigenetics describes how social experience modulates the body at the genetic level (Chung et al 2016; Rose \& Abi-Rached 2013). New biological understandings of 'the social life of the brain' are being used to animate policies and practices in healthcare, education and other social domains (Pickersgill 2013: 322), with sociological debate consequently focusing on how 'the webs of human social and cultural life that we had come to understand as our particular object of knowledge seem more and more open to being figured neuroscientifically' (Fitzgerald \& Callard 2015: 4). In this vein, there have been calls to 'develop concepts and methods for understanding and describing biological forms of human life that emerge within, and are reproduced by, specific kinds of social, political and economic relations' (Fitzgerald et al 2016: 16).

Biosocial assessments of neuroscience in particular have paved the way for 'critical neuroscience' that focuses on the implications of the 'brain facts' produced by neuroscience for social practices and power relations (Williams et al 2011). Critical neuroscience depicts how 'neuroscientific objects of inquiry [are...] held in place by a number of factors coproduced by a collection of circumstances, social interests, and institutions' (Choudhury \& Slaby 2012: 31). These approaches take seriously the biological materiality of the body while simultaneously advancing understandings of power and 'the social' as dynamic and complex (Pitts-Taylor 2016). This is opposed to existing supposedly interdisciplinary endeavours such 
as social or cultural neuroscience which deploy binary, static and restricted accounts of how social, cultural and environmental phenomena affect neurobiology (Fitzgerald \& Callard 2015). In this paper we further distinguish between forms of biosocial knowledge which assume the centrality of biology to social explanation, and those which critically interrogate the governance implications of new forms of neuroscientific expertise, authority and power.

Biosocial explanations derived from epigenetics and brain science are now beginning to be applied to expand neuroscientific understanding of education and learning (Asbury and Plomin, 2014; Blakemore \& Frith 2005; Mareschal et al 2013; Szucs and Goswami 2007). The field of 'neuroeducation' is notable as both an interdisciplinary academic development (educational neuroscience) and a diverse set of school-based practices, such as brain-targeted teaching and learning and cognitive enhancement, which reflect 'the dispersal of neurobiological language, imagery, symbolism and rhetoric within formal and informal learning environments' (Busso \& Pollack 2015: 169). As Costandi (2016: 4) notes, neuroplasticity has become a 'buzzword' while the phrase 'rewire your brain' has become a 'mantra' evoked 'by educationalists and business managers in their attempts to enhance learning and improve leadership skills.' In some cases, within the neuroeducational discourse, a machinic conception of the brain and a computational account of learning is quite explicit. Davidson (2011:14-15), for example, likens the brain to an iPhone, 'with apps for just about everything':

Those apps can be downloaded or deleted and are always and constantly in need of a software update. ... The brain is similar. How we use our brain ... changes our brain. ... In this way the iPhone also corresponds nicely with recent advances in what we know about neural plasticity, the theory that the brain adapts physically to the sensory stimuli it receives.

Pykett (2015: 97) has critiqued the tendencies in neuroeducation 'to reduce learning to an algorithmic or computational process' - as is particularly the case with the multimillion dollar industry in 'brain training' apps and games (Costandi 2016). As such, the possibilities and limits of neuroscience in education have been the focus of a burgeoning critical scholarship (Varma et al. 2008; Immordino-Yang 2011; Carrasco et al. 2015). Critical studies have examined the rise of neuroeducation and epigenetics, and the specifically reductive notions of the social and environmental determinants of learning and development on which they have been based (Baker 2015; de Vos 2014; Gillborn 2016). Bruer (1997) has argued ideas of brain-based approaches to education are often grounded on misconceptions and overgeneralizations about neuroscience. Consequently, Purdy and Morrison (2009: 108) call 
for a 'critical filter' to prevent unchallenged applications of neuroscientific claims to education, while Busso and Pollack (2015) argue that neuroscientific discourses have the power to reconfigure identities and reframe how individuals make sense of the goals, purposes, and values of schooling.

Meanwhile a number of scholars have also adopted biosocial accounts of childhood in an attempt to move beyond apparently outmoded social constructivist theorizations of the child. Prout (2005) rejects dualisms in favour of the multiple materialities which shape contemporary childhood - including information technologies, genetics and psychopharmaceuticals — while Lee and Motzkau (2013: 192) have described 'biosocial tweaking' as the process of 'making adjustments at the level of life processes,' such as the use of neuro-linguistic programming, as 'biotechnical fixes' to produce 'socially preferred abilities and qualities' in children. Likewise, Kraftl (2013: 18) 'seeks an expanded sense of the constituents in relations between and beyond human beings - of, for instance, the role of neuroscientific chemical processes in intergenerational relations between parents/carers and children.' Youdell (2016a) draws on these hybrid conceptualizations to propose that biosocial theories might change our existing understandings of the learning process itself:

as the interaction between a person and a thing; as embedded in ways of being and understanding that are shared across communities; as influenced by the social and cultural and economic conditions of lives; as involving changes to how genes are expressed in brain cells because it changes the histones that store DNA; as provoking certain parts of the brain into electrochemical activity; as relying on a person being recognised by others, and recognising themselves, as someone who learns. Shared meanings, gene expression, electrochemical signals, the everyday of the classroom and a sense of self are all ... parts of the phenomenon that is learning. (Youdell 2016a: 5)

This revised neurobiologically and epigenetically informed conception of learning aims 'to engage with the human as a biocultural creature, bringing together the intra-acting forces of culture and biology' (Youdell 2016b: 799). However, the neurocomputational trends that we recount suggest there remains significant critical work to be done to understand the potential effects of digital technologies as they have been woven into current enthusiasm for the biosocial within education.

\section{Programming behaviours}


As social and cultural environments become increasingly digitized, computer technologies need to be considered as part of the biosocial mix. A recent 'foresight' report produced by the Human Brain Project documents many recent developments in neuroscience, artificial intelligence, cognitive computing, and other neurotechnologies of machine learning and deep learning that use the brain as inspiration:

The power of these innovations has been increased by the development of data mining and machine learning techniques, that give computers the capacity to learn from their 'experience' without being specifically programmed, constructing algorithms, making predictions, and then improving those predictions by learning from their results, either in supervised or unsupervised regimes. In these and other ways, developments in ICT and robotics are reshaping human interactions, in economic activities, in consumption and in our most intimate relations. (Rose et al 2016: 5)

Other studies have emphasized how an ongoing 'neurotechnology revolution' in brainmachine interface and neuroapplication development is producing new 'brain-computersociety entanglements' that challenge existing legal and ethical systems and pose risks for neural security and mental privacy (Ienca \& Andorno 2017: 5). Neurocomputation is not, however, a new field of enquiry. Among its learned societies and publications, the International Neural Network Society was established in 1987 and its journal Neural Networks in 1988, whilst the Neural Computation journal was founded in 1989. A multitude of research studies have explored various aspects of learning including memory and adaptive processes from a neurocomputational perspective. The neurocomputational perspective that sees cognition itself as a biologically realistic learning algorithm is now being brought to bear on the development of new educational technologies.

As a framework for making sense of the landscape of emerging neurocomputation and its consequences for educational governance and learning, we advance the idea of 'brain/code/space'. The original idea of 'code/space' we have adapted has been influentially detailed by geographers Kitchin and Dodge (2011) to describe how 'software creates new spatialities of everyday life and new modes of governance':

Code/space occurs when software and the spatiality of everyday life become mutually constituted, that is, produced through one another. Here, spatiality is the product of code, and the code exists primarily to produce a particular spatiality. ... Any space that is dependent on software-driven technologies to function as intended constitutes a code/space. (Kitchin \& Dodge 2011: 16-17)

Ultimately, code/spaces are programmable environments, in that code fundamentally alters how a software-driven space functions and is experienced. Importantly, the relationship 
between software and space is neither deterministic nor universal, but rather 'how code/space emerges through practice is contingent, relational and context dependent' as well as embodied in the 'unpredictable interactions ... between people and code' (Kitchin \& Dodge 2011: 18). Nonetheless, for Kitchin and Dodge, software is now transforming the means by which individuals and societies may be governed. Historically, the management of modern societies has depended on the production of systems to collect, collate and analyse information about populations, social institutions and businesses. This particular 'biopolitical' style of governance depends on viewing people as parts of larger systems, whose actions and behaviours might therefore be monitored as citizens with rights and obligations to the state, or as problems to be solved and whose behaviours might be targeted for management, intervention and alteration (Foucault 2008).

The rise of software systems leads to a new mode of biopolitical governance which Kitchin and Dodge (2011: 85) term 'automated management':

Automated management is the regulation of people and objects through processes that are automated (technologically enacted), automatic (the technology performs the regulation without prompting or direction), and autonomous (regulation, discipline and outcomes are enacted without human oversight). Software controls ... systems that actively reshape behaviour by altering the performance of a task.

Code/spaces are part of a new mode of technologically-enacted biopolitical governance in which software participates as an automated, automatic and autonomous actor in the surveillance and structuring of human behaviour and action. Technological environments have, in other words, become key techniques for monitoring and managing society as a whole by regulating people to comply with the specific criteria of behaviour coded-in to software. Code/spaces are simultaneously governed by those who program them to perform preset functions, and enact governing techniques to compel people to comply with specific encoded behavioural defaults and norms.

Programmable environments that might alter behaviours constitute part of what we designate as brain/code/space, but we also include conceptualizations of how digital technologies are designed for more explicit purposes of behaviour change emerging from behaviour science and its associated technical field of persuasive computing. Behavioural science has become attractive to governments as a way of intervening in people's lives to change their behaviours in particular 'desirable' ways. The 'behaviour change agenda' is based on the assumption that human behaviour is largely habitual and predictable, and therefore open to modification, and has 'led to the gradual formation of new governmental programmes that seek to guide 
and compensate for the behaviours of the sub-optimal citizen' (Jones et al 2013: 3). The dominant idea in the fast-growing 'behaviour change' agenda is that of the 'nudge' - the idea that designing 'choice architectures' (or the varied physical, sociocultural and administrative environments in which choices are framed) can alter or activate people's behaviours in a predictable way (Thaler and Sunstein 2008).

Recently, specifically brain-based techniques of governance and policy approaches to behaviour change have been documented. Pykett (2015: ix) claims that:

efforts to map the brain, to capitalise on the vast datasets emerging from contemporary neuroscience, and eventually to develop the computing power to simulate neural functioning are the latest indications of a culture in which the brain is privileged in its explanatory power for all manner of human experiences, decisions, capabilities, actions and relationships.

By exploring the intersections of political, economic and social practice with specific brain claims and brain-based activities, Pykett (2015) shows how a 'brain culture' is re-shaping contemporary practices of governance, public policies and social practices in varied environments including those of the workplace, social care, and education. In other words, new knowledge, expertise, representations of and metaphors derived from the brain as the supposed source of human behaviour are being used in new kinds of policy experimentation. This represents a new kind of biopolitics of the brain sciences as 'governing the conduct of human beings [has] come to require, presuppose and utilize a knowledge of the human brain' (Rose and Abi-Rached 2014: 3-5). Behaviour science and neuroscience are now increasingly informing educational policy as a means of promoting socially desired behaviours among young people (McGimpsey et al 2016). As Pitts-Taylor $(2016: 18,39)$ argues, neuroscientifically based training programs, curricula and pedagogies that 'target the brain for modification or rewiring' represent strategies of 'preemptive neurogovernance' that work with the plasticity of the brain for individual, societal and economic improvement.

The brain-based behaviour change techniques that have emerged from hybridizing 'nudge theory' with neuroscientific insights into behaviour change are increasingly intersecting with software development to create new brain/code/spaces of governance. Expert knowledges about how to nudge people's behaviours have been explicitly coded in to software in the field of 'persuasive computing,' which focuses on the design of machines to influence human thinking and behaviors across domains including health, business, safety, and education (Fogg 2002). In the era of persuasive computing, behavioural nudging can be understood as software-driven 'hypernudges,' or 'algorithmic decision-guidance techniques' that can alter 
people's behaviour in a predictable way (Yeung 2017). As persuasive technologies flood into physical spaces through devices and interfaces, new kinds of behaviour-changing code/spaces are created.

Media theorist Hayles (2017) has particularly noted the implications of such technologies for understanding human cognition, arguing that cognition may in fact be located in technical systems rather than solely in the mental world of an individual participant. Her nonanthropocentric view of 'cognition everywhere' suggests that recent advances in computing mean machines can use their experiences to learn, achieve skills and interact with people. When these nonconscious cognitive devices inhabit social environments and penetrate into human systems, she claims, they can then potentially modify the dynamics of human behaviours through changing brain morphology and functioning. The potential of nonhuman neurocomputational techniques based on the brain, then, is to become legible as traces in the neurological circuitry of the human brain itself, and to impress itself on the cerebral lives and identities of citizens.

Crucially for our conception of brain/code/space, there is a 'circularity effect' of neuro governance, as neuroscientific 'knowledges about the brain become the basis of social intervention, public policy, popular culture and everyday practice, and thus feed back into our understandings of the "self" which is the object of so much neuroscientific endeavour' (Pykett 2015: 64). This notion resonates with Hayles' (2017: 115) contention that technical systems have rendered brain-like cognition to exist in nonhuman machines in ways which in turn reshape brain function and morphology, thereby producing 'human-technical cognitive assemblages' that consist of both 'human and nonhuman cognizers' in distributed cognitive systems.

In the term brain/code/space, we therefore capture a specific mode of neurocomputational governance whereby behaviours may be altered by automated machines that have been programmed to function in ways that emulate some aspects of the human brain and cognition, and which therefore transform the functioning of the spaces in which they are embedded. These machines are brain-targeted and designed to interact with human users to alter, enhance and extend their cognitive capacities. As with the original concept of code/space we have adapted, there is nothing deterministic or universal about the brain/code/spaces produced when technical systems are embedded in environments to form cognitive assemblages with human cognizers. Without wishing to overdetermine the capacity of 
neurotechnologies to influence human actions, behaviours and cognitions — or to ignore their potential to generate advantages for society—we view brain/code/space as the hybrid progeny of computational systems and brain-based behaviour change agendas that are increasingly inhabiting and animating spatial environments. Brain/code/space enacts a dynamic neurocomputational biopolitics, a mode of governance that relies on neuroscientific technologies to provide evidence and insight about the brain as a way of understanding people's behaviour, and that deploys brain-based technologies as a means to interact with individuals and 'hypernudge' their behaviours, actions, and even cognition itself. Notably, these technologies are primarily being developed and promoted by commercial organizations, in ways that suggest new forms of neurocomputational capital are being assembled and distributed as these organizations and their consumers seek comparative advantage by inserting their technologies in diverse contexts, such as healthcare, finance and education.

In what follows, we detail how global technology and publishing firms are constructing new kinds of artificially intelligent and cognitive learning environments in education. These educational brain/code/spaces exemplify emerging neurocomputational techniques of both brain-inspired and brain-inspiring governance that are being designed to be enacted across other spatial locations including cities, offices, healthcare systems and other networked cognitive systems. We offer a critical biosocial perspective that is attentive to the ways in which sociotechnical code/space environments might become legible as traces in behaviours, bodies and brains.

\section{Pearson, AI \& human capital development}

Pearson has been promoting itself as a new source of expertise in educational data analysis since establishing its Center for Digital Data, Analytics and Adaptive Learning and its global Learning Curve databank in 2012. The ambitions of this centre were to use the masses of data becoming available as educational activities increasingly occur via digital media, to derive new theories of learning processes and cognitive development (Williamson 2016). The Learning Curve gathers this data as a way of constructing a global index of 'country performance in education,' measured and ranked in terms of 'cognitive skills.' Together, the Learning Curve and Pearson's support for educational big data analyses propose to make diverse forms of data collected at both the scale of the individual and the population into new evidentiary sources to guide policy decision-making (Hogan, Sellar \& Lingard 2016). 
Pearson has taken its work on big data further in recent proposals to implement artificial intelligence in education. Pearson's report, Intelligence Unleashed: An argument for AI in education proposes that artificial intelligence can transform teaching and learning:

\footnotetext{
Although some might find the concept of AIEd alienating, the algorithms and models that comprise AIEd form the basis of an essentially human endeavour. AIEd offers the possibility of learning that is more personalised, flexible, inclusive, and engaging. It can provide teachers and learners with the tools that allow us to respond not only to what is being learnt, but also to how it is being learnt, and how the student feels. (Luckin et al 2016: 11)
}

Pearson proposes a 'marketplace' of thousands of AI components combining to 'enable system-level data collation and analysis that help us learn much more about learning itself and how to improve it' (12). In technical terms, what Pearson terms AIEd consists of data analytics techniques, machine learning algorithms, computer modelling, statistics, artificial neural networks and neuroscience, since 'AI involves computer software that has been programmed to interact with the world in ways normally requiring human intelligence. This means that AI depends both on knowledge about the world, and algorithms to intelligently process that knowledge' (18). Pearson therefore envisions algorithmically intelligent educational systems as code/spaces where 'humans live and work alongside increasingly smart machines' (12).

Pearson's application proposals include Intelligent Tutoring Systems which 'use AI techniques to simulate one-to-one human tutoring, delivering learning activities best matched to a learner's cognitive needs and providing targeted and timely feedback, all without an individual teacher having to be present' (24). It also proposes intelligent support for collaborative working — such as AI agents that can integrate into teamwork - as well as intelligent virtual reality environments that simulate authentic contexts for learning tasks, with teachers supported by their own AIEd teaching assistants and AIEd-led professional development programs.

Pearson's account of intelligent, personalized learning environments is therefore based on new understandings of 'how to blend human and machine intelligence effectively' (30), which assume specific disciplinary models of human intelligence and cognition:

\footnotetext{
AIEd will continue to leverage new insights in disciplines such as psychology and educational neuroscience to better understand the learning process, and so build more accurate models that are better able to predict - and influence - a learner's progress, motivation, and perseverance. ... Increased collaboration between education neuroscience and AIEd developers will provide technologies that can
} 
offer better information, and support specific learning difficulties that might be standing in the way of a child's progress (37).

Insights from neuroscience will be translated into models that can be used to predict and intervene in individuals' learning processes through AIEd systems. In particular, this highlights Pearson's ambition to mobilize AIEd applications in the development of 'human capital.', with metric accounts of learners' cognitive skills positioned as proxy indicators of national progress in producing productive economic contributors of the future. RobertsMahoney et al (2016: 2) suggest that commercial edu-businesses such as Pearson are seeking to apply 'big data'-based methods in ways which:

\footnotetext{
position teaching and learning within a reductive set of economic goals and purposes that emphasize human capital development and training future workers ... [and] advocate for the expansion of datadriven instruction and decision-making, while conceptualizing learning as the acquisition of discrete skills and behavior modification detached from broader social contexts and culturally relevant forms of knowledge and inquiry.
}

The brain-based techniques and applications of AIEd are seen by Pearson as ways of modelling learners' cognition in order to build human capital through an educational environment re-constructed as a neurocomputational code/space:

Once we put the tools of AIEd in place as described above, we will have new and powerful ways to measure system level achievement. ... AIEd will be able to provide analysis about teaching and learning at every level, whether that is a particular subject, class, college, district, or country. This will mean that evidence about country performance will be available from AIEd analysis, calling into question the need for international testing. (Luckin et al 2016: 48)

In other words, Pearson is proposing to turn educational systems at large into networked brain/code/spaces where neuro-based technologies will perform constant measurement and management of learning environments and of all those individuals who inhabit them. It emphasizes the role of neurocomputation in the performativity of education systems, as brain-inspired technologies that can optimize human capital development and simultaneously enact intelligent real-time measurement at large scale to produce data which might be collated into global comparative databanks — such as Pearson's own proprietary Learning Curve.

\section{IBM, cognitive systems \& rewiring the brain}


IBM has taken inspiration from neuroscience even further in its cognitive computing systems for education. One of the world's most successful computing companies, IBM has recently turned its attention to educational data analytics:

Analytics translates volumes of data into insights for policy makers, administrators and educators alike so they can identify which academic practices and programs work best and where investments should be directed. By turning masses of data into useful intelligence, educational institutions can create smarter schools for now and for the future. (IBM 2013)

According to its paper, Personalized Education: from curriculum to career with cognitive systems (King et al 2016), IBM's 'cognitive learning systems' approach is based on neuroscientific methodological innovations, neuro-anatomy, technical developments in braininspired (super)computing, new programming language, artificial neural networks algorithms and cognitive system applications. Over the last decade, IBM has positioned itself as a dominant research centre in cognitive computing, with huge teams of engineers and computer scientists working alongside in-house neuroscience experts on both basic and applied research in this area. Its research activities are underpinned by claims that cognitive computing will 'emulate the human brain's abilities for perception, action and cognition.' IBM has dedicated extensive R\&D to the production of 'neurosynaptic brain chips' and scalable 'neuromorphic systems'-hardware designed to emulate the neural network processes of the brain in silicon-as well as its cognitive supercomputing system Watson. Based on this program of work, in its white paper Computing, cognition and the future of knowing, IBM defines cognitive systems as 'systems that learn at scale, reason with purpose and interact with humans naturally. Rather than being explicitly programmed, they learn and reason from their interactions with us and from their experiences with their environment' (Kelly 2015: 2).

To apply its cognitive computing applications in education, IBM has developed a specific Cognitive Computing for Education program. Its program director has presented its intelligent, interactive systems that combine neuroscientific insights based on 'using supercomputers to understand cognitive processes such as learning' with technologies that can 'learn and interact with humans in more natural ways':

At the intersection of cognitive neuroscience and cognitive computing lies an extraordinary opportunity ... to refine cognitive theories of learning as well as derive new principles that should guide how learning content should be structured when using cognitive computing based technologies. (Nitta 2015) 
The prototype innovations developed by the program include automated 'cognitive learning content', 'cognitive tutors' and 'cognitive assistants for learning' that can understand the learner's needs and 'provide constant, patient, endless support and tuition personalized for the user.' IBM imagines that 'deeply immersive interactive experiences with intelligent tutoring systems can transform how we learn,' ultimately leading to the 'utopia of personalized learning':

Until recently, computing was programmable - based around human defined inputs, instructions (code) and outputs. Cognitive systems are in a wholly different paradigm of systems that understand, reason and learn. In short, systems that think. What could this mean for the educators? We see cognitive systems as being able to extend the capabilities of educators by providing deep domain insights and expert assistance through the provision of information in a timely, natural and usable way. (King et al. 2016: 9)

Rather than being hard-programmed, cognitive computing systems are thus designed like the brain to learn from experience and adapt to environmental stimuli, in ways that resonate with contemporary biosocial conceptualizations of epigenetics and neuroplasticity. IBM sees cognitive systems as optimizing and enhancing the role of the teacher, as a kind of cognitive prosthetic or machinic extension of human qualities. This is part of a historical narrative about human-computer hybridity that IBM has wrapped around its cognitive computing R\&D:
Across industries and professions we believe there will be an increasing marriage of man [sic] and machine that will be complementary in nature. This man-plus-machine process started with the first industrial revolution, and today we're merely at a different point on that continuum. At IBM, we subscribe to the view that man plus machine is greater than either on their own. (10)

These claims register with longer-term interests across the cognitive sciences and neuroscience in an extended, embedded, embodied and enacted conception of the mind which considers cognition itself more as an ecological system than as property internal to the cranium. In turn, these interests reflect a longer historical narrative of human-machine hybridity by which formerly accepted boundaries 'between carbon-based organic components and silicon-based electronic components' have been erased by a 'posthuman' view which 'configures human being so that it can be seamlessly articulated with intelligent machines' (Hayles 1999: 2-3).

The focus on cognition as a 'brain-body-environment system' underpins IBM's ambitions. The promise of cognitive computing for IBM is not just of more 'natural systems' with 'human qualities,' but a fundamental reimagining of the 'next generation of human cognition, 
in which we think and reason in new and powerful ways,' as claimed its white paper Computing, cognition and the future of knowing: 'It's true that cognitive systems are machines that are inspired by the human brain. But it's also true that these machines will inspire the human brain, increase our capacity for reason and rewire the ways in which we learn' (Kelly 2016: 11). A recursive relationship between machine cognition and human cognition is assumed in this statement. It sees cognitive systems as both brain-inspired and brain-inspiring, both modelled on the brain and remoulding the brain through interacting with users.

IBM's R\&D in cognitive computing fundamentally depends on its own in-house neuroscientific findings about neuroplasticity, and the translation of the biological neural networks studied in computational neuroscience into the artificial or convolutional neural networks used in applied cognitive computing and AI research. It is, in other words, deploying its own kind of biosocial explanations about (1) the functioning of the plastic brain, (2) the possibility of emulating the brain in silicon, and (3) about the amenability of the brain to be 'rewired' through interaction with nonhuman cognitive systems. Supported by evidence about epigenetics and the plasticity of the brain from its in-house neuro-experts, IBM's cognitive computing engineers have constructed systems that emulate the brain as a networked device constantly adapting to environmental input. In these ways, technologies such as cognitive systems for education can be seen as techniques of governance, insofar as they are based on claims to an objective knowledge about human embrained nature that can be used to underpin interventions to target human cognition and behaviour. Moreover, IBM proceeds from a deficit view of cognition, maintaining that human cognition and intelligence can and should be enhanced and extended — by being rewired — through its integration with machine-based cognitive systems.

Caution is required, however about these deterministic assumptions that cognitive systems will inevitably lead to brain rewiring. IBM's Watson has recently been subjected to criticism that its machine learning capacities have been over-exaggerated through marketing (Freedman 2017). An ‘imaginary of plasticity’ underpins its ambitions, which promotes the idea that the brain is flexible, malleable, mouldable, able to be trained, rewired, improved and ultimately optimized (Rose \& Abi-Rached 2013). Such an imaginary has inspired governmental programs, commercial brain-based products, as well as brain-targeted pedagogic initiatives and technologies, and needs to be understood in terms of a wider governmentality of personal improvement and self-optimization: 
There is clearly an 'elective affinity' ... between this emphasis on plastic, flexible brains and more general sociopolitical changes that prioritize individual flexibility across the life span to accommodate to rapidly changing economic demands, cultural shifts, and technological advances — and that demand a constant labor of self-improvement on the part of today's citizens. (Rose \& Abi-Rached 2013: 223)

IBM, like Pearson and other edu-businesses, is thoroughly engaged in a reimagining of the capacities, skills and dispositions required of young people in a period of significant technological and economic change. As stated in the introduction to IBM's report on cognitive systems in education:

There is a growing disconnect between what education delivers and the skills being demanded in today's ever-changing global marketplace. The net result is that upon leaving full-time education, many young people are ill-prepared for the world of work. At the same time, we are seeing unprecedented levels of change across industries and professions, with digital technologies serving as agents of transformation. (King et al 2016: 2).

For IBM, the capacity for human capital development and the production of labour for this technological context lies in the brain itself. Through its explorations in neuroscience, it is claiming and marketing its authority to know and be able to intervene in cognitive processes by activating the brain itself using advanced cognitive systems that have been designed to integrate themselves into human cognition and from there to enhance and optimize it as appropriate to contemporary demands.

\section{Biosocial brain/code/space}

Late in 2016, IBM and Pearson announced a global partnership which would involve embedding IBM's Watson technologies in Pearson's courseware content at massive scale. Together, they are seeking to operationalize their shared imaginary of embedding a more cognitive or intelligent system for enhancing human cognition and capital into educational environments. We can begin to understand what Pearson and IBM are proposing in the light of emerging biosocial explanations and their application to emerging forms of neurocomputation. To some extent, Pearson and IBM are themselves mobilizing biosocial explanations - as distinct from critical biosocial theory - in the development of their techniques and applications. Pearson's AIEd intelligent learning environments and IBM's cognitive classroom are reconfigured as neurocomputationally 'brainy spaces' in which learners are targeted for cognitive enhancement and neuro-optimization through interacting with other nonconscious cognitive agents and intelligent environments. In this way, brain- 
based machine intelligences are proposed to enmesh with the human brain, influencing brain morphology and cognitive functioning. Through the marketing visions and investments of the likes of Pearson and IBM, the artificially intelligent, cognitive educational environment is translated into what we have articulated as biosocial brain/code/space, wherein cognitive hybrids of human and nonhuman cognition may be shaped to fit particular commercial, educational and governance objectives. Brain/code/space describes brain-based techniques of behaviour change that are deployed through software to create new kinds of social environments that might govern the activities of those who occupy such spaces.

The main critical issues here are how the learning brain is represented and modelled by organizations such as Pearson and IBM as well as how neurocomputational forms of capital circulate within and beyond the classroom reframed as a cognitive learning system in some quite specific ways. In the same manner that the field of neuroeducation has been criticized for representing the brain as a multiply connected device that might be debugged, rewired and optimized, these practices of neurocomputation tend towards a reductive and algorithmic account of education as a matter of machinic learning. This reflects a longstanding tendency, rooted in 1940s cybernetics and cognitivism, by scientists and technologists to liken the functions of the brain to the operations of a computer, with the role of physical hardware played by the brain itself and thoughts serving as software (Epstein 2016).

The information-processing metaphor of the brain matters because it assumes that neural functioning — and by default processes of learning — can be computationally modelled to construct technologies that are taken to have 'natural' and 'human' qualities of learning and cognition. Cognitive science has repeatedly utilized metaphors of computing for the functions and processes of the brain — such as that the brain is hardware, a rapid, complex calculating machine, made up of digital switches; that the mind is an information machine, or a software program which manipulates symbolic representation; that thinking is computation; that memory is looking up stored data; and that the function of the mind and brain is information processing (Edwards 1997: 161). It follows from these computing metaphors that the complexity of the human brain, processes of thinking and the production of behaviour can therefore be reduced to the relatively simpler processes of computer programming, with behavioural 'bugs' and thought patterns viewed as amenable to being de-bugged, reprogrammed or re-wired (Edwards 1997). 
In the context of brain plasticity, a whole range of techniques and neurotechnologies 'now target the brain for modification and rewiring' for purposes of neurogovernance (Pitts-Taylor 2016: 18). IBM's claims that its cognitive systems behave in more 'natural' ways inspired by neuroplasticity - rather than being hard-programmed in the dominant style of computing that has existed since the 1940s — advance a new computing metaphor of the brain as networks of neural patterns connected to a world of information and communication networks (Castells 2009: 139). In contrast to characterizations of cognition as an input-processing-output model, or the brain as a standalone information-processing device, IBM represents the brain as a complex networked platform that is multiply connected, adaptive, and constant 'rewiring' itself according to environmental input and experiential stimuli received from a vast ecosystem of both human and nonhuman cognitive platforms. A plausible model of neural structure and functioning based on the 'brain-facts' of plasticity and epigenetics underpins its cognitive systems, but this is a model produced via a distinctive disciplinary style of thinking that imagines computing and cognition can be understood in synonymous terms. Hayles (2017: 25) argues 'it should not be surprising' that both human and technical cognitive systems 'share attributes in common, because brains ... designed them'-but the brains of these designers are themselves situated in technical settings with their own socialized worldviews, systems of thinking, and ways of conceptualizing cognition as a computational process.

This matters because as those technologies are put into contact and interaction with learners, they are intended to reshape and rewire cognitive capacities in ways that are consonant with the computational model. As Hayles (2017) notes, when cognitive devices inspired by neuroplasticity penetrate into human systems, they can potentially modify the dynamics of human behaviours through changing brain morphology and functioning. Hayles (2017: 119) describes 'human-nonhuman cognitive assemblages' in which human cognition and technical cognition interpenetrate and affect the other, bringing about 'neurological changes in the mindbodies of users.' From a more overtly neuroscientific position, Costandi (2016: 99) similarly acknowledges that some forms of 'brain training' can and do 'affect brain structure and function.' Clearly, the cognitive technologies produced by IBM for deployment into specifically educational spaces have the potential to interact with human cognizers, produce classroom cognitive assemblages, and shape the neurobiological correlates of cognition in learners. 
However, IBM is seeking to shape the neurobiological correlates of human cognition in particular ways informed both by its computational understanding of cognition and by its vision of activating human capital through nonhuman enhancement of human cognition. It, like cognitive science before it, treats cognition as if it can be debugged and optimized like software. As with other neurotechnology developments inspired by the brain, humans are conceived 'as mere components in complex ICT and robotics systems, components which might be optimized and interfaced in the same terms as the silicon parts', raising the risk that the affective, interpersonal and social capacities of the human brain may be treated in rationalistic terms as an information processor, with the aim to model essential aspects of mental processes to eventually replace them in a quest for optimization (Rose et al. 2016: 22). IBM's ideal that cognitive systems can 'rewire the ways we learn' inscribes the computational networking metaphor onto the plasticity of the learning brain, reimagining the basis, purpose and the governance of educational institutions in the process. While the systems that IBM and Pearson are promoting may be plausibly brain-based then, they reproduce a problematic understanding of mental life in terms of computational information processing and networking platforms. Specifically, they conflate the neurobiological neural networks of the brain that are the products of neuroscientific imaging and basic research with the artificial neural networks that can be modelled through mathematical operations and are being developed in applied R\&D in AI and cognitive computing. They not only treat the neural networks of the learning brain as computable, but may be privileging brain-inspired machine learning models over the complexity of embodied, culturally relevant and socially situated learning.

In so doing, their constructions of the classroom as a cognitive learning system not only downplay the social as a mode through which learning takes place and by which educational difference plays out. So too they initiate new distributions of neurocomputational capital by which global technology companies and publishing firms control educational big data, as well as the capacity, algorithms and proprietary systems through which such data can be analysed. Their aspirations to rewire human cognition itself through neuroenhancement also raise wider concerns about the power available to states and companies to use neurotechnology coercively to manipulate the embrained mental states of citizens (Ienca \& Andorno 2017). In this context, the emphasis of Pearson and IBM on using neurotechnologies to activate the capacities appropriate to the development of human capital is part of wider governmental and commercial strategies of neurogovernance that locate 
behaviours and cognitions in the brain, and seek to modify their actions by targeting them for neurological activation and enhancement (Pitts-Taylor 2016). The emergence of brain/code/space constitutes a spatial enactment of neurogovernance strategies whereby individuals may be coercively assembled into human-technical cognitive hybrids in order to achieve commercial aspirations around human capital optimization.

\section{Conclusion}

While we need to be cautious of the extent to which neurocomputational developments might (or might not) actually occur (or be desirable), it is important to analyse them as part of a growing interest in how technologically-enhanced social environments based on the brain might interweave with the neurobiological mechanisms that underlie processes of learning and development — whether as intended or not. In other words, Pearson's and IBM's application of AI and cognitive systems to education need to be interpreted as biosocial matters of significant contemporary concern.

Indeed a kind of biosocial enthusiasm about the plasticity of the brain and epigenetic modulation animates the technological ambitions of Pearson and IBM. In this paper, we have begun to outline the emergence of AIEd and cognitive systems in education specifically as a biosocial phenomenon which actively creates new kinds of educational spaces in which human and nonhuman cognizers form hybrid cognitive assemblages. We have described the ways that the brain is being understood, modelled, simulated and integrated into the kinds of AI applications and cognitive systems that such companies are promoting. We have offered the analytical framework of brain/code/space to elaborate how emerging neurocomputational forms of governance might reshape educational spaces and rework the idea of the learner though both brain-based and brain-targeted interventions. We have detailed the processes by which this can be achieved, including the deployment of neuroscientific insights, imaginaries of neuroplaciticity and epigenetics, and metaphors of the brain used in the development of machine learning and cognitive learning systems. Though we acknowledge the potential transformative effects on human cognition as it is interpenetrated by technical cognition to produce distributed and enhanced forms of networked cognition, we remain concerned about neurogovernance techniques that privilege computational models of the brain and seek to rewire plastic brains to fit particular commercial and governmental aspirations. 
The translation of educational environments into biosocial spaces that are technologically enhanced by new forms of AI, cognitive systems and other neurocomputational applications could have far-reaching effects on teachers, learners, educational institutions and social relations. And yet as with emerging practices of neuroeducation, very little is known about the current uptake, distribution and potential consequences of neurocomputational governance in education. In order to understand this complex phenomenon our starting point has been to trace the neurocomputational imaginaries invested in such spaces, and to begin interrogating the kinds of human-technical cognitive hybrids that might result. A critical biosocial perspective on education offers a useful and timely basis for future investigations of just how particular distributions of neurocomputational capital and forms of biopolitical governance might be developing within this new brain/code/space.

\section{References}

Asbury, K \& Plomin, R. 2016. G is for Genes: The Impact of Genetics on Education and Achievement. Chichester: Wiley-Blackwell.

Baker, B. 2015. From 'Somatic scandals' to 'a constant potential for violence'? The culture of dissection, brainbased learning, and the rewriting/rewiring of 'the child.' Journal of Curriculum and Pedagogy 12(2): 168-197.

Blakemore, S.J. \& Frith, U. 2005. The Learning Brain: Lessons for Education. Oxford: Blackwell.

Bruer, J. T. 1997. Education and the brain: a bridge too far. Educational Researcher 26(8): 4-16.

Busso D, Pollack C. 2015. No brain left behind: Consequences of neuroscience discourse for education. Learning, Media, and Technology 40(2):168-186.

Carrasco, J. G., Serrano, M. J. H. \& Garcia, A. V. M. 2015. Plasticity as a framing concept enabling transdisciplinary understanding and research in neuroscience and education. Learning, Media and technology 40(2): 152-167.

Castells, M. 2009. Communication Power. Oxford: Oxford University press.

Choudhury, S. and Slaby, J. (eds) 2012. Critical neuroscience: A handbook of the social and cultural contexts of neuroscience. Chichester: Wiley-Blackwell.

Costandi, M. 2016. Neuroplasticity. London: MIT Press.

Davidson, C. 2011. Now You See It: How the brain science of attention will transform the way we live, work and learn. London: Viking.

de Vos, J. 2016. The death and the resurrection of (psy)critique. The case of neuroeducation. Foundations of Science 21(1): 129-145.

Edwards, P.N. 1997. The Closed World: Computers and the politics of discourse in Cold War America. London: MIT Press.

Epstein, R. 2016. The empty brain. Aeon, 18 May 2016: https://aeon.co/essays/your-brain-does-not-processinformation-and-it-is-not-a-computer

Fitzgerald, D. and Callard, F. 2015. Social science and neuroscience beyond interdisciplinarity: experimental entanglements. Theory, Culture and Society 32(1): 3-32. 
Foucault, M. 2008. The Birth of Biopolitics: Lectures at the Collège de France, 1978-1979. Edited by M. Senellart, trans. G. Burchell. New York: Palgrave Macmillan.

Freedman, D.H. 2017. A reality check for IBM's AI ambitions. MIT Technology Review, 27 June: https://www.technologyreview.com/s/607965/a-reality-check-for-ibms-ai-ambitions/

Gillborn, D. 2016. Softly, Softly: Genetics, Intelligence and the Hidden Racism of the New Genism. Journal of Education Policy 31 (4): 365-388.

Hayles, N.K. 1999. How We Became Posthuman: Virtual bodies in cybernetics, literature and informatics. London: University of Chicago Press.

Hayles, N.K. 2017. Unthought: The power of the cognitive nonconscious. London: University of Chicago Press.

IBM. 2016. Personalized Education: from curriculum to career with cognitive systems. Portsmouth: IBM Corporation.

Ienca, M. \& Andorno, R. 2017. Towards new human rights in the age of neuroscience and neurotechnology. Life Sciences, Society \& Policy 13(5): 1-27.

Immordino-Yang, M. H. 2011. Implications of affective and social neuroscience for educational theory. Educational Philosophy and Theory 43(1): 98-103.

Kelly III, J.E. 2015. Computing, cognition and the future of knowing: How humans and machines are forging a new age of understanding. Somers, NY: IBM Corporation.

King, M., Cave, R., Foden, M. \& Stent, M. 2016. Personalised education: From curriculum to career with cognitive systems. Portsmouth: IBM Corporation.

Kitchin, R. \& Dodge, M. 2011. Code/Space: Software and everyday life. London: MIT Press.

Kraftl, P. 2013. Beyond 'voice', beyond 'agency', beyond 'politics'? Hybrid childhoods and some critical reflections on children’s emotional geographies. Emotion, Space and Society 9: 13-23.

Lee, N. \& Motzkau, J. 2013. Tweak: Biosocial imaginations and educational futures. In N. Selwyn and K. Facer (eds) The Politics of Education and Technology: 191-208. New York: Palgrave Macmillan.

Luckin, R., Holmes, W., Griffiths, M. \& Forcier, L.B. 2016. Intelligence Unleashed: An argument for AI in education. London: Pearson.

Mareschal, D. Butterworth, B. \& Tolmie, A. (eds) 2013. Educational neuroscience. Chichester: John Wiley \& Sons.

Meloni, M., Williams, S. \& Martin, P. 2016. The biosocial: sociological themes and issues. Sociological Review Monograph Series: Biosocial Matters: Rethinking Sociology-Biology Relations in the Twenty-First Century, 64, no. 1: 7-25.

Pitts-Taylor, V. 2016. The Brain's Body: Neuroscience and corporeal politics. London: Duke University Press.

Prout, A. 2005. The future of childhood. London: Routledge Falmer.

Purdy, N. \& Morrison, H. 2009. Cognitive neuroscience and education: unravelling the confusion. Oxford Review of Education 35(1): 99-109.

Pykett, J. 2015. Brain Culture: Shaping policy through neuroscience. Bristol: Policy Press.

Pykett, J. \& Disney, T. 2015. Brain-targeted teaching and the biopolitical child. Politics, Citizenship and Rights. http://dx.doi.org/10.1007/978-981-4585-94-1_22-1

Roberts-Mahoney, H. Means, A.J. \& Garrison, M.J. 2016. Netflixing human capital development: personalized learning technology and the corporatization of K-12 education. Journal of Education Policy:

http://dx.doi.org/10.1080/02680939.2015.1132774 
Rose, N., Aicardi, C. \& Reinsborough, M. 2016. Future computing and robotics: A foresight report from the Human Brain Project lab. London: King's College London

Szucs, D., and Goswami, U. 2007. Educational neuroscience: Defining a new discipline for the study of mental representations. Mind, Brain, and Education 1: 114-127.

Varma, S., McCandliss, B., and Schwartz, D. 2008. Scientific and pragmatic challenges for bridging education and neuroscience. Educational Researcher 37(3): 140-152.

Williams, S., Katz, S. \& Martin, P. 2011. The neuro-complex: some comments and convergences. Media Tropes 3, no. 1: 135-146.

Williamson, B. 2016. Digital methodologies of education governance: Pearson plc and the remediation of methods. European Educational Research Journal 15, no. 1: 34-53.

Williamson, B. 2017. Computing brains: learning algorithms and neurocomputation in the smart city. Information, Communication and Society 20, no. 1: 81-99.

Yeung, K. 2016. Hypernudge: big data as a mode of regulation by design. Information, Communication and Society 20, no. 1: 118-136.

Youdell, D. 2016a. A biosocial education future? Research in Education: http://rie.sagepub.com/content/early/2016/09/16/0034523716664579

Youdell, D. 2016b. New biological sciences, sociology and education. British Journal of Sociology of Education 37(5): 788-800. 